\title{
Origin of Discrepancies in Inelastic Electron Tunneling Spectra of Molecular Junctions
}

\author{
Lam H. Yu, ${ }^{1}$ Christopher D. Zangmeister, ${ }^{1}$ and James G. Kushmerick ${ }^{1, *}$ \\ ${ }^{1}$ National Institute of Standards and Technology, Gaithersburg, MD 20899
}

(Dated: November 20, 2018)

\begin{abstract}
We report inelastic electron tunneling spectroscopy (IETS) of multilayer molecular junctions with and without incorporated metal nano-particles. The incorporation of metal nanoparticles into our devices leads to enhanced IET intensity and a modified line-shape for some vibrational modes. The enhancement and line-shape modification are both the result of a low lying hybrid metal nanoparticle-molecule electronic level. These observations explain the apparent discrepancy between earlier IETS measurements of alkane thiolate junctions by Kushmerick et al. [Nano Lett. 4, 639 (2004)] and Wang et al. [Nano Lett. 4, 643 (2004)].

PACS numbers: 73.63.-b, 73.63.Rt, 73.40.Gk, 73.21.-b
\end{abstract}

Inelastic electron tunneling spectroscopy (IETS) is unique in that it provides an in situ vibrational probe of a molecular electronic junction. The IET spectrum represents a molecular signature for a junction, simultaneously proving that the molecule of interest is present and giving insight into how the charge carriers interact with the molecule 11]. Experimental IET spectra can be analyzed with the aid of recently developed computational techniques [2, 3, 4] to yield information on the nature of the metal-molecule interfaces [5], the orientation of the molecules $[6,7]$, and the position of the molecules relative to the metallic electrodes [8]. With further refinements to the interpretive modeling tools, IETS has the potential to become a standard characterization technique for all molecular electronic devices. However, recent IETS measurements of alkenethiolates in two different test beds, crossed-wire [9] and nanopore 10] junctions, have yielded markedly different spectra. The most obvious differences between these spectra are their different lineshapes and the presence or absence of the $\mathrm{C}-\mathrm{H}$ stretching modes. While it was originally proposed that the discrepancy in observing the $\mathrm{C}-\mathrm{H}$ stretching modes resulted from differences in the two molecules studied, namely a monothiol in the crossed-wire experiment and a dithiol in the nanopore experiments, it has subsequently been demonstrated both experimentally [11] and theoretically [12] that this difference alone is not sufficient to account for the observed discrepancies.

In this letter, we report IET spectra of multilayer junctions with and without incorporated metal nanoparticles (Figure 1). The incorporation of metal nanoparticles $(<$ $1 \mathrm{~nm}$ diameter) with low lying electronic states into a large energy gap $(>2 \mathrm{eV})$ molecular layer can lead to what we coin enhanced IETS. The experimental manifestations of enhanced IETS include orders of magnitude increase in the IET spectral intensity and drastic changes in the lineshapes of select vibrational modes.

Fabrication of molecule-particle-molecule (MPM) multilayer devices [27] is based on previous work done on $\mathrm{Cu}-$ dithiol [13, 14] and Ni-dithiol[11] multilayer thin films. Cartoons illustrating the structures of the MPM mul-
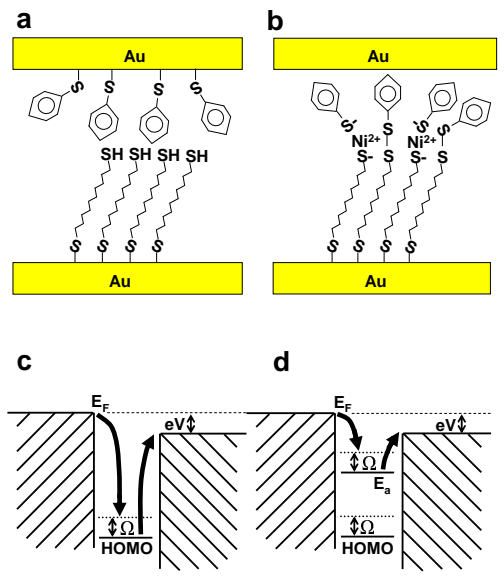

FIG. 1: (Color online) Structural illustration of (a) the control device, where a monolayer of BT and $\mathbf{C 1 0}$ is self-assembled onto either electrode, and (b) the molecule-particle-molecule device, where layers of $\mathbf{C 1 0}$ and $\mathrm{Ni}$ and $\mathbf{B T}$ are sequentially self-assembled onto one electrode. Energy diagram for inelastic electron tunneling via (c) a molecular orbital of the control device, and (d) a hybrid metal particle-molecule electronic resonance level, $E_{a}$, in the molecule-particle-molecule device. ( $E_{F}$ is the Fermi energy, $\Omega$ is the energy of a vibration mode, and $\mathrm{eV}$ is the applied bias.)

tilayer and the control device consisting of a benzenthiol (BT) monolayer on one electrode and a 1,10decanedithiol (C10) monolayer on the other electrode (without the incorporation of metal particles) are shown in Fig. 1. Details of the crossed-wire tunnel junction apparatus used in our experiments have been previously reported [9, 11, 15]. The crossed-wire apparatus is housed in a stainless steel vacuum chamber that is evacuated and purged with He gas before being submerged into a liquid He storage dewar. All transport measurements are made after the crossed-wire junction has been immersed in liquid He for at least $1 \mathrm{~h}$. Transport measurements are performed with standard ac modulation techniques with two lock-in amplifiers that record the first and second harmonics signals (proportional to $d I / d V$ and $d^{2} I / d V^{2}$, respectively) simultaneously with the I-V char- 


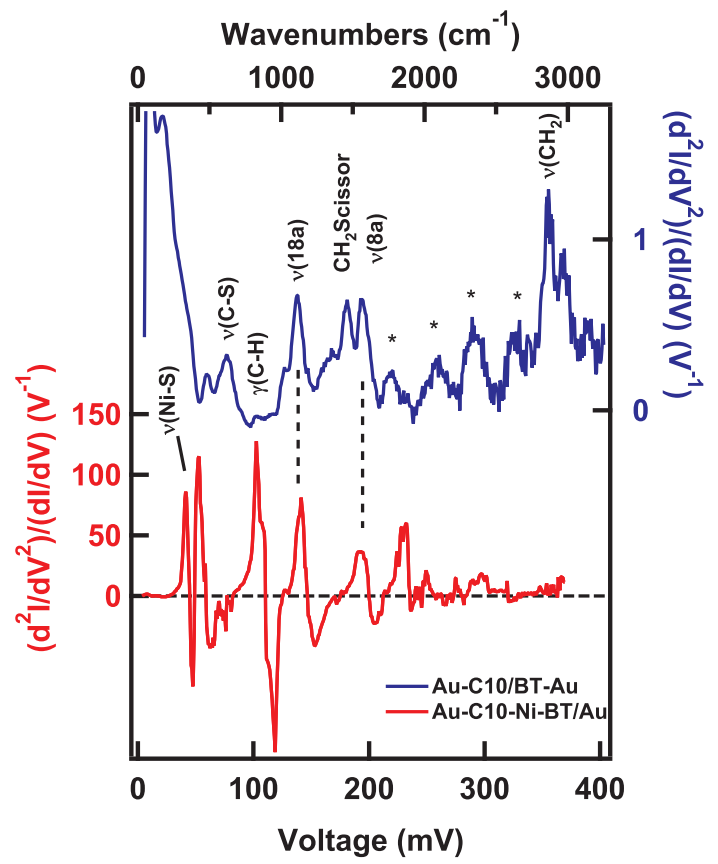

FIG. 2: (Color online) IET spectra of a C10-Ni-BT multilayer junction (bottom) and a C10/BT control junction (top). Mode assignments are based on previous experimental results and theoretical calculations. Note $v=$ stretch, $\gamma=$ out of plane bend and the phenyl modes are given in terms of Wilson-Varsanyi terminology. The asterisks mark modes likely associated with the free thiol group of the C10 layer. Modes associated with the metal nanoparticles and the BT layer dominate in the C10-Ni-BT junction.

acteristics. The amplitude of the ac modulation used is $4 \mathrm{mV}$ RMS. To remove any dependence on the junction area, the spectra are presented as the normalized amplitude $\left(d^{2} I / d V^{2}\right) /(d I / d V)$.

Figure 2 shows the IET spectra of a MPM junction and that of a control junction. Since these junctions contain both aliphatic and aromatic molecules, the observed spectra exhibit vibrational modes associated with both moieties. We assign the observed features to specific molecular vibrations by comparison to previous IETS results [5, 9, 11] and density functional theory calculations. Although there are a number of similarities between the spectra of the two junctions, there are clear differences as well. The normalized amplitude of the MPM junction IETS features are enhanced by over two orders of magnitude compared with those of the control junction. In addition to the enhancement there is a clear change in the peak shape. While all the observed spectral features of the control junction have a simple peak shape, the vibrational features in the MPM junction have distinctive peak-derivative-like lineshapes. Such intensity enhancement and peak-derivative lineshapes are never observed in IET measurements on non-metal embedded crossed-wire junctions [9, 11], nor in magnetic bead junctions [5]. Another striking difference between the two spectra in Fig. 2 is that the aliphatic $\mathrm{C}-\mathrm{H}$ stretching mode near $360 \mathrm{mV}$ is missing in the MPM junction spectrum. Similarly, the zero-bias feature (ZBF) 28] which extends out to $\approx 50 \mathrm{mV}$ in the control junction is almost imperceptible in the MPM junction spectrum. A change of scale (not shown) reveals that the MPM junction does in fact contain a ZBF that extends out to $\approx 30 \mathrm{mV}$ with a normalized amplitude of $\approx 1.5 \mathrm{~V}^{-1}$, comparable in absolute magnitude to that of the control device ZBF.

Figure 1; and 1d show schematically how the embedment of metal particles in a molecular junction can give rise to a metal particle-molecule hybrid resonant energy level close to the Fermi level, which could leads to the enhancement of the IET signal. In the control device, the molecular vibrational modes are coupled to the molecular orbitals of the constituent molecules, but in the MPM junctions the molecular vibrational modes are also coupled with a new hybrid level which lies much closer to the Fermi level than the highest occupied molecular orbital (HOMO) of the constituent molecular layers. With a resonant level close to the Fermi level, higher order elastic cotunneling processes can contribute substantially to the electronic transport near the onset of an inelastic channel[16, 17, 18, 19]. In a low-order perturbative treatment of this effect, Baratoff and Persson found that a resonant level close to the Fermi level could lead to larger IETS intensity and substantial modification of the spectral lineshapes [16, 17]. According to this perturbative model and more advanced theoretical treatments based on the non-equilibrium Green's function (NEGF) formalism [19, 20], the manner in which the intensity and lineshapes of the IET spectrum of our MPM junctions will be modified depends on the energy, $E_{a}$, and width, $\Gamma$, of the metal particle-molecule hybrid resonance, and on its coupling to the molecular vibration, $\delta E$. The electronphonon coupling term, $\delta E$, describes the shift of $E_{a}$ under the influence of the excitation of a particular vibrational mode.

Baratoff and Persson find that the relative change in conductance, $d \sigma / \sigma$, upon reaching the threshold for excitation of a molecular vibration, $|e V|=\Omega$, is proportional to

$$
\frac{\delta E^{2}}{\left(E_{a}-\overline{E_{F}}\right)^{2}+(\Gamma / 2)^{2}} \frac{\left(\overline{E_{F}}+\Omega-E_{a}\right)^{2}-(\Gamma / 2)^{2}}{\left(\overline{E_{F}}+\Omega-E_{a}\right)^{2}+(\Gamma / 2)^{2}},
$$

where $\overline{E_{F}}=E_{F}-e V$, with $E_{F}$ being the Fermi level of the unbiased electrodes, $e$ is the charge of an electron, $V$ is the applied bias and $\Omega$ is the energy of the vibrational mode [16, 17]. They show that, depending on the position and width of the metal particle induced electronic level, the normalized amplitude of the IETS feature can be very large, and the lineshape can be a peak, a peakderivative, or a dip. The numerical modeling results of Galperin et al. clearly demonstrate the evolution of a 


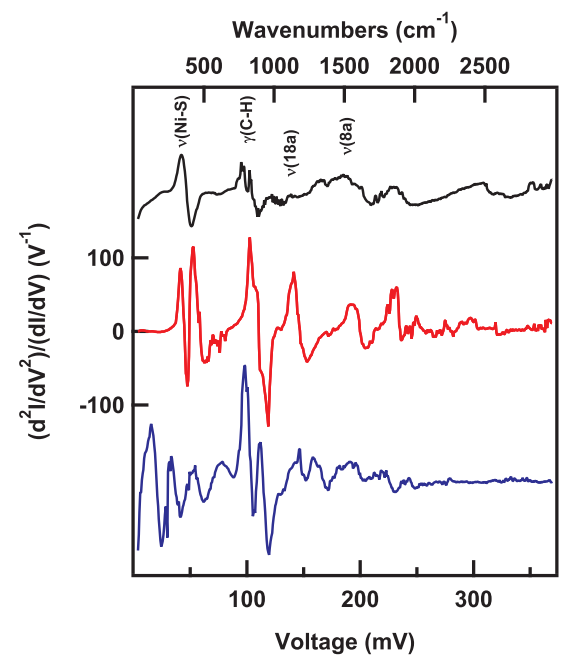

FIG. 3: (Color online) IET spectra of three different C10Ni-BT multilayer junctions (spectra are offset vertically for clarity). The mode assignments are the same as those in Figure 2

IETS feature from a peak to a peak-derivative to a dip as the position of the electronic resonance level shifts (see Fig. 1 of Ref. 19).

An alternative mechanism that could give rise to the modified IET spectra observed in the MPM junctions is resonant tunneling through vibronic states of the molecular layer. The signature of such a process is equally spaced conductance peaks (or peak-derivative features in the $d^{2} I / d V^{2}$ spectrum), where the energy spacing between the features corresponds to a fundamental vibrational mode of molecular layer 21, 22]. Unlike the IETS process we described above which involves virtual excitation of vibronic states, this resonant process involves a real transition to vibronic states. While we cannot rule out resonant vibronic excitation as the origin of the observed modified features, there are two characteristics of our data which make such interpretation difficult to justify. First, we note the peak-derivative features are not evenly spaced. An analysis of the distribution of energy spacings between features in our MPM data reveals that successive features are separated from one another by one of three different energy spacings roughly, $17 \mathrm{mV}$, $33 \mathrm{mV}$ and $56 \mathrm{mV}$. Furthermore, the onset of features in our MPM junction occurs at bias $<40 \mathrm{mV}$. For the resonant tunneling interpretation to be correct the metal level would need to be practically degenerate with the Fermi level of the electrodes 29].

Figure 3 shows the IET spectra of three different MPM junctions (The middle trace is the same as the MPM spectrum shown in Fig. 21). While the same spectral features dominate each spectrum, there is substantial variation in the normalized amplitude and the lineshapes between the three spectra. The bottom two spectra are $\approx$ 3.5 times more intense than the top spectrum. In light of

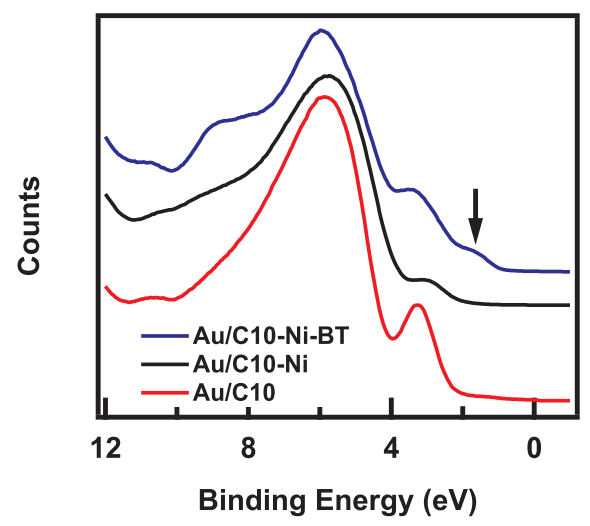

FIG. 4: (Color online) Ultraviolet photoelectron spectra of $\mathrm{Au} / \mathbf{C 1 0}, \mathrm{Au} / \mathbf{C 1 0}-\mathrm{Ni}$, and $\mathrm{Au} / \mathbf{C 1 0}-\mathrm{Ni}-\mathbf{B T}$ (spectra are offset vertically for clarity). The arrow points to the hybrid electronic resonance arising from the metal coordination in the $\mathrm{Au} / \mathbf{C 1 0}-\mathrm{Ni}-\mathbf{B T}$ monolayer. Binding energies are referenced as positive for occupied states below the Fermi level (binding energy $=0 \mathrm{eV}$ ). UP spectra were obtained with excitation via a He I line source $(21.2 \mathrm{eV})$ with a hemispherical electrostatic analyzer (resolution $\approx 100 \mathrm{meV}$ ).

Eq.1, such device to device variations of IET spectrum intensity is not surprising since $E_{a}, \Gamma$, and $\delta E$ depend strongly on the specific nanoscale environment of each device.

The presence of a low lying electronic resonance in our MPM junctions is experimentally confirmed by ultraviolet photoelectron spectroscopy (UPS). Figure 4 shows the UP spectra of a $\mathrm{Au} / \mathbf{C 1 0}$ (bottom), a $\mathrm{Au} / \mathrm{C10}-\mathrm{Ni}$ (middle), and a $\mathrm{Au} / \mathbf{C 1 0}-\mathrm{Ni}-\mathrm{BT}$ (top) molecular layer. In UPS, one-photon photoelectron processes are employed to provide direct measurement of the energies and characteristics of the occupied electronic levels of the assembled molecular layer of interest. The most intense peak on all three spectra at $\approx 5.9 \mathrm{eV}$ is attributed to the sigma bonds of the alkane backbone 23]. The peak at $3.2 \mathrm{eV}$ in the $\mathrm{Au} / \mathrm{C10}$ molecular film is attributed to the localized 3p lone-pair electrons on the top-terminal $\mathrm{S}$ of the C10 molecules [23]. In the $\mathrm{Au} / \mathbf{C 1 0}-\mathrm{Ni}$ film this feature is attenuated and shifted to a lower binding energy of $\approx$ $3 \mathrm{eV}$, consistent with the coordination of the $\mathrm{Ni}$ atoms to the terminal $\mathrm{S}$. The shoulder feature at $\approx 3.3 \mathrm{eV}$ on the $\mathrm{Au} / \mathbf{C 1 0}-\mathrm{Ni}-\mathrm{BT}$ spectrum is actually a convolution of the localized 3p S lone-pairs and the HOMO of the benzenethiol molecule 24].

In contrast, the HOMO of the $\mathrm{Au} / \mathbf{C 1 0}-\mathrm{Ni}-\mathrm{BT}$ molecular layer is characterized by the shoulder structure centered at $\approx 1.5 \mathrm{eV}$. The UP spectrum of a control molecular layer composed of $\mathrm{Au} / \mathbf{C 1 0}-\mathrm{Ni}-\mathbf{C 6}$ (not shown), where C6 represents 1-hexanethiol, shows no energy level near $1.5 \mathrm{eV}$. This strongly suggests that this low lying electronic state is a delocalized Ni-BT hybrid energy level. This interpretation is further supported by the observa- 
tion that the $\mathrm{Ni}$ and $\mathbf{B T}$ vibrational modes are the only modes enhanced in the C10-Ni-BT junction (Figs. 2 and 3).

From the UPS data we extract $E_{a}$ for the MPM junctions as $1 \mathrm{eV}$ based on the onset energy of the hybrid energy level. An inspection of the IETS literature suggests that $\delta \mathrm{E}$ ranges from $0.3 \mathrm{eV}$ to $0.4 \mathrm{eV}$ and $\Gamma$ ranges from $0.8 \mathrm{eV}$ to $1 \mathrm{eV}[16,18,20]$. Using these parameters and Eq. 1, a rough estimate of $\mathrm{d} \sigma / \sigma$ for the $\nu(8 \mathrm{a})$ stretching mode at $\approx 185 \mathrm{meV}$ of the MPM junctions is $2 \%$ to $9 \%$. The experimental value of $\mathrm{d} \sigma / \sigma$ for this vibrational mode as extracted from the spectra shown in Fig. 3 is $(7$ $\pm 3) \%$. For comparison the experimental value of $\mathrm{d} \sigma / \sigma$ for this same vibraional mode in the control junction is $0.2 \%$. The agreement between the estimated and experimental $d \sigma / \sigma$ strongly suggests that the essential physics of resonantly enhanced IETS is captured by Eq. 1 and the formalism from which it is derived.

Based on the data presented here we feel that it is likely that the nanopore devices of Wang et al. contained nanoscale metal islands within the 1,8-octanedithiol monolayer [10]. Incorporation of metal islands - most likely formed during the top contact deposition [25] would explain the lineshapes they observed as well as the lack of $\mathrm{C}-\mathrm{H}$ stretching mode. More recent IET spectra of nanopore devices with ferromagnetic electrodes and 1,8octanedithiol molecules 26] again showed characteristics consistent with metal nanoparticle incorporation.

In summary, we observed that the incorporation of metal nanoparticles into molecular junctions can result in substantial modification to the intensity and lineshape of the IET spectrum. By comparing the IET spectra of our MPM crossed-wire junctions with those from nanopore devices, we conclude that the embedment of metal nanoparticles into the molecular layer of the nanopore devices is the likely cause of the differences previously observed in crossed-wire 9 and nanopore 10 junctions of similar molecular systems.

The authors thank L. Richter for useful discussions. Financial support from the DARPA MoleApps program is gratefully acknowledged.

* Electronic address: james.kushmerick@nist.gov

[1] A. Troisi and M. A. Ratner, Small 2, 172 (2006).

[2] A. Troisi and M. A. Ratner, Physical Review B 72, 033408 (2005).

[3] N. Sergueev, D. Roubtsov, and H. Guo, Physical Review Letters 95, 146803 (2005).

[4] G. C. Solomon, A. Gagliardi, A. Pecchia, T. Frauenheim, A. Di Carlo, J. R. Reimers, and N. S. Hush, Journal of Chemical Physics 124, 094704 (2006).

[5] D. P. Long, J. L. Lazorcik, B. A. Mantooth, M. H. Moore, and M. A. Ratner, Nature Materials 5, 901 (2006).

[6] M. Kula, J. Jiang, and Y. Luo, Nano Letters 6, 1693 (2006).
[7] A. Troisi and M. A. Ratner, Nano Letters 6, 1784 (2006).

[8] M. Galperin, A. Nitzan, M. A. Ratner, and D. R. Stewart, Journal of physical Chemistry B 109, 8519 (2005).

[9] J. G. Kushmerick, J. Lazorcik, C. H. Patterson, R. Shashidhar, D. S. Seferos, and G. C. Bazan, Nano Letters 4, 639 (2004).

[10] W. Wang, T. Lee, I. Kretzschmar, and M. A. Reed, Nano Letters 4, 643 (2004).

[11] L. H. Yu, C. D. Zangmeister, and J. G. Kushmerick, Nano Letters 6, 2515 (2006).

[12] J. Jiang, M. Kula, W. Lu, and Y. Luo, Nano Letters 5, 1551 (2005).

[13] M. Brust, P. M. Blass, and A. J. Bard, Langmuir 13, 5602 (1997).

[14] T. L. Brower, M. Cook, and A. Ulman, Journal of Physical Chemistry B 107, 11721 (2003).

[15] J. G. Kushmerick, D. B. Holt, J. C. Yang, J. Naciri, M. H. Moore, and R. Shashidhar, Physical Review Letters 89, 086802 (2002).

[16] B. N. J. Persson and A. Baratoff, Physical Review Letters 59, 339 (1987).

[17] A. Baratoff and B. N. J. Persson, Journal of Vacuum Science and Technology A 6, 331 (1987).

[18] T. Mii, S. G. Tikhodeev, and H. Ueba, Physical Review B 68, 205406 (2003).

[19] M. Galperin, M. A. Ratner, and A. Nitzan, Nano Letters 4, 1605 (2004).

[20] M. Galperin, M. A. Ratner, and A. Nitzan, Journal of Chemical Physics 121, 11965 (2004).

[21] H. Sumi, Journal of physical Chemistry B 102, 1833 (1998).

[22] X. H. Qiu, G. V. Nazin, and W. Ho, Physical Review Letters 92, 206102 (2004).

[23] D. M. Alloway, M. Hofmann, D. L. Smith, N. E. Gruhn, A. L. Graham, J. Colorado, R., V. H. Wysocki, T. R. Lee, P. A. Lee, and N. R. Armstrong, Journal of physical Chemistry B 107, 11690 (2003).

[24] C. D. Zangmeister, S. W. Robey, R. D. van Zee, N. E. Gruhn, Y. Yao, and J. M. Tour (2007), manuscript in preparation.

[25] A. V. Walker, T. B. Tighe, J. J. Stapleton, B. C. Haynie, S. Upilli, D. L. Allara, and N. Winograd, Applied Physics Letters 84, 4008 (2004).

[26] W. Wang and C. A. Richter, Applied Physics Letters 89, 153105 (2006).

[27] MPM multilayer thin films are constructed by first immersing a $\mathrm{Au}$ wire into a $3 \mathrm{mM}$ ethanol solution of 1,10decanedithiol for $\approx 14 \mathrm{~h}$. The wire is sonicated in fresh ethanol for 5 min to remove any physisorbed molecules, then placed in a $35 \mathrm{mM} \mathrm{Ni}\left(\mathrm{ClO}_{4}\right) 2 * 6 \mathrm{H}_{2} \mathrm{O}$ ethanol solution for $3 \mathrm{~h}$. Following the thiol reaction with the $\mathrm{Ni}$ and another $5 \mathrm{~min}$ of sonication, the wire is exposed to a $3 \mathrm{mM}$ solution of benzenethiol in ethanol for $14 \mathrm{~h}$ then sonicated for $5 \mathrm{~min}$.

[28] The origin of the ZBF has been discussed previously 9 , 11] and is believed to originate from phonon interactions in the gold wires.

[29] Although we do not attribute the overall spectra to a Frank-Condon progression expected for the resonant tunneling mechanism it is possible that the strong feature at $\approx 100 \mathrm{mV}$ is a combination of the second harmonic of the $\mathrm{Ni}-\mathrm{S}$ vibration and the phenyl $\mathrm{C}-\mathrm{H}$ out of plane bending mode 20]. 